\title{
The Obsessive Compulsive Scale of the Child Behavior Checklist predicts obsessive- compulsive disorder: a receiver operating characteristic curve analysis
}

\author{
James J. Hudziak, ${ }^{1}$ Robert R. Althoff, ${ }^{1}$ Catherine Stanger, ${ }^{1}$ \\ C.E.M. van Beijsterveldt, ${ }^{2}$ Elliot C. Nelson, ${ }^{4}$ Gregory L. Hanna, ${ }^{3}$ \\ Dorret I. Boomsma, ${ }^{2}$ and Richard D. Todd ${ }^{4}$ \\ ${ }^{1}$ Department of Psychiatry, University of Vermont, Burlington, USA; ${ }^{2}$ The Free University, Amsterdam, The \\ Netherlands; ${ }^{3}$ Department of Psychiatry, University of Michigan, Ann Arbor, USA; ${ }^{4}$ Departments of Psychiatry and \\ Genetics, Washington University School of Medicine, St. Louis, Missouri, USA
}

\begin{abstract}
Background: The purpose of this study was to determine a score on the Obsessive Compulsive Scale (OCS) from the Child Behavior Checklist (CBCL) to screen for obsessive compulsive disorder (OCD) in children and to rigorously test the specificity and sensitivity of a single cutpoint. Methods: A receiver operating characteristic (ROC) curve analysis was applied to data from 61 patients with clinically determined OCD, 64 clinical controls and 73 general population controls to determine the best sum score on the CBCL-OCS to predict confirmed OCD in children. Using the ROC-determined cutoff, this score was applied to a national sample of CBCL data from 2460 singleton children ages 4-18 and to 20,016 children ages 7-18 from three large general population twin samples to determine the estimated prevalence in the general population. Results: Using a CBCL-OCS score of 5 demonstrated an area under the curve (AUC) of .88 with high sensitivity (92\%) and moderate specificity (67\%) compared to clinical controls. Compared to the general population controls, the AUC was .96 with high sensitivity $(92 \%)$ and specificity (89\%). In the twin samples, the number of participants with CBCL-OCS scores above this cutpoint was $2.3-7.1 \%$. Conclusions: These findings suggest that the OCS of the CBCL may provide a highly effective way to screen for childhood OCD, and that the prevalence of childhood OCD may have been underestimated, thus prompting the need for further research into screening children for this condition. Keywords: Obsessive compulsive disorder, Child Behavior Checklist, prevalence, Obsessive Compulsive Scale.
\end{abstract}

Concerns that the prevalence of obsessive compulsive disorder (OCD) is higher than previously thought in children (Flament et al., 1988; Zohar, 1999; Zohar et al., 1992) have fueled the need to find sensitive and specific instruments for screening the pediatric population to identify children at risk. While the prevalence of OCD in younger children remains relatively unstudied, most estimates place the number between .13\% and .25\% (Costello et al., 1996; Steinhausen, Metzke, Meier, \& Kannenberg, 1998), although Costello et al., 1996 used the externalizing score of the CBCL to screen for OCD. The prevalence is thought to increase throughout childhood (Heyman et al., 2001) until reaching the adult prevalence of $1-3 \%$ by late adolescence (Snider $\&$ Swedo, 2000). Nearly $80 \%$ of adult OCD cases have their onset before age 18 (Pauls, Alsobrook, Goodman, Rasmussen, \& Leckman, 1995).

However, children experiencing subclinical symptoms may be as high as 19\% (Valleni-Basile et al., 1994) and cases of OCD in childhood often are not diagnosed until the child's behavior disrupts family life (long after symptoms began) (Calvocoressi et al., 1995). Given this, and the secrecy inherent in OCD
(Rapoport et al., 2000), there is need for a more quantitative approach to screening for OCD. Because there are effective medication (Grados, Scahill, $\&$ Riddle, 1999) and psychotherapeutic (Piacentini, 1999; March, Franklin, Nelson, \& Foa, 2001; March $\&$ Mulle, 1998) treatments for OCD, early identification and treatment should clearly be an objective.

A contributing factor in the underdiagnosis of OCD is that pediatricians and family practitioners may have neither received training nor had experience in identifying OCD in children. It has been estimated that only $10 \%$ of cases of childhood OCD are identified by primary care physicians (Riddle, 1998). In our prior work we addressed the many obstacles to screening for OCD in epidemiologic studies. Although each of the diagnostic instruments for OCD has many strengths, their use in prior epidemiologic research has been complicated by the need for expert clinicians to perform the interview, the length of the interviews, and in some cases the validity of the interviews (Nelson et al., 2001). The screening tools for childhood OCD [e.g., The Leyton Obsessional Inventory - Child Version (Berg, Rapoport, \& Flament, 1985), Yale Brown Obsessive Compulsive 
Scale - Child Version (YBOCS) (Goodman et al., 1989)] are not commonly used in primary care offices. In order to effectively screen for OCD in a variety of clinical settings, a screening approach must be short (clinicians are faced with severe time constraints), valid, sensitive and specific, useful for children of all ages and both genders, and be easy to explain to parents and children alike.

Using data from the Childhood Behavior Checklist (CBCL) (Achenbach, 1991), we have developed a scale that can be used to identify children with OCD. In our earlier work (Nelson et al., 2001), we identified 11 of the 119 CBCL items as being consistent with the behaviors of childhood OCD. We then used factor analysis to test and derive an Obsessive Compulsive Scale (OCS) from the $11 \mathrm{CBCL}$ items. A single factor accounted for $40.0 \%$ of the variance and, after simplification of the solution, consisted of 8 items. We then compared $\mathrm{CBCL}$ weighted factor scores for a cohort of pediatric OCD patients who had been diagnosed as having DSM-IV OCD with scores from clinically ascertained and general population controls. We demonstrated that the OCS from the CBCL had moderate sensitivity (75.3-84.9\%) and high specificity (82.2-92.5\%), with positive predictive value (PPV) ranging between $70.5 \%$ and $83.3 \%$ and negative predictive value (NPV) ranging between $88.2 \%$ and $91.6 \%$. These findings have since been independently validated by Geller and colleagues (Geller, Coffey, Doyle, Shaw, \& Biederman, 2003).

However, our initial study and the work by Geller had a number of limitations. First, it is unrealistic to expect a clinician to calculate weighted factor scores in order to obtain a score on the CBCL-OCS. Second, because the CBCL-OCS was tested on such a small sample, it was unclear what the endorsement rates of the CBCL-OCS items would be in the general population. We wanted to examine whether use of a simple addition of these item scores would yield accurate detection of OCD. This study did so using receiver operating characteristic (ROC) analyses.

ROC analyses allow visual analysis of the relative merits of continuous scales as predictors of dichotomous outcomes. ROC analyses have been used for diverse purposes ranging from predicting response to pharmacological interventions to determining predictors of violent recidivism (Rey, Morris-Yates, \& Stanislaw, 1992; Rice \& Harris, 1995). ROC analysis provides a means to assess the predictive value of a given test and to adjust cutpoints for clinical or research purposes. On the ROC graph, the sensitivity of different cutpoints on the test are graphed on the $Y$ axis (true positive rate) along with 1 minus the specificity (1 - specificity) of the cutpoints on the $\mathrm{X}$ axis (false positive rate) to determine the ability of the test to optimize both measures for each point on the test. The higher the graph extends toward the upper left corner of the graph, the higher the discriminatory power of the test. The area under the curve (AUC) statistic assesses the probability of correctly classi- fying a randomly selected pair of subjects in which one is a case and one is a noncase. AUC values range between .5 , in which correct classification occurs in $50 \%$ of cases, and 1.0, in which correct classification occurs with every case (sensitivity and specificity = $100 \%)$.

This study proceeded in two steps. First, we used an ROC analysis applied to a sample of clinically referred children who met DSM criteria for OCD, a sample of clinically referred children who did not meet DSM criteria for OCD, and general population controls to determine an acceptable clinical cutpoint. Next, this cutpoint was applied to a general population sample of children and to two large general-population twin samples to determine the percentage of children who may benefit from further screening in the general population.

\section{Methods}

\section{Participants and procedure}

Data on children and adolescents were derived from four sources. For determining the accuracy characteristics of the CBCL-OCS using the ROC, CBCL data from patients with clinical OCD, non-OCD clinical controls, and matched general population controls were examined. This clinical population has been described previously (Nelson et al., 2001). Briefly, 73 patients with clinical OCD were identified from patients who presented for treatment to St. Louis Children's Hospital Child Psychiatry Center, the UCLA Neuropsychiatric Institute, and the University of Michigan. Diagnoses were made using DSM-IIIR rules by board-certified psychiatrists with clinical expertise in the diagnosis and treatment of OCD. Psychiatrically ill clinical controls were obtained from the St. Louis Children's Hospital Child Psychiatry Center. These participants were selected using a computer program that generated random numbers and then attempted to match the clinical controls to an OCD group member in terms of age (within a year), sex, and self-described ethnic/racial group. If an OCD individual could not be readily matched using this method, a billing record search was conducted to locate a clinical control for that participant. Clinical control participants were excluded if no CBCL was available or if there was clear documentation of OCD symptoms in their record. Of particular note is that both the clinical controls and the OCD group were selected from a highly comorbid population. As noted in Nelson et al. (2001), about $21 \%$ of the OCD group also had ADHD, 26\% also had affective disorders, and 25\% also had other anxiety disorders. The clinical controls had significantly more affective disorders $(40 \%)$, disruptive disorders $(32 \%)$, and ADHD (47\%). Seventythree general population non-clinical controls were obtained using the $1991 \mathrm{CBCL}$ national normative sample which has been described elsewhere (McConaughy, Stanger, \& Achenbach, 1992). Participants were chosen who matched an OCD patient in terms of sex, age, and race. Participants were excluded if CBCL data on any of the 8 items of the CBCL-OCS were missing. This excluded 12 OCD patients and 9 clinic 
controls, leaving 61 and 64 participants, respectively. None of the general population controls were excluded. CBCL-OCS scores were computed for each of these individuals and the results were placed into the ROC analysis.

After determining the cutoff level for the CBCL-OCS, this cutoff was applied to CBCL data from nonhandicapped, nonclinically referred participants taken from the 1991 national normative sample as a whole (Achenbach, 1991). Briefly, these data were obtained from home interview surveys with the parents of participants chosen to be representative of the contiguous 48 states. These surveys included the CBCL and other questions regarding demographics and the participant's mental health and special education history. We started with a sample of 2110 children. Participants were excluded if any of the $8 \mathrm{CBCL}-\mathrm{OCS}$ items were not completed. CBCL-OCS scores from each of the 2101 participants remaining were calculated and frequency tables were prepared. Because CBCL norms have been determined separately for boys and girls and in age groups between 4-11 and 12-18, frequency tables were determined separately for these four groups. The mean age of boys was $7.8(\mathrm{SD}=2.2)$ for the $4-11$ age group and $14.5(\mathrm{SD}=1.8)$ for the $12-18$ age group. The mean age of girls was $7.7(\mathrm{SD}=2.2)$ for the $4-11$ age group and $14.2(\mathrm{SD}=1.6)$ for the $12-18$ age group.

Because many of the research applications of the CBCL-OCS for modeling OCD will be determined using large twin samples, we next applied this screening tool to two large general-population twin samples. First, we used twins from part of an ongoing project, the Missouri Twin Study. This sample has been previously described (Hudziak et al., 1998). Briefly, parents of all twins born in Missouri between 1975 and 1991 (assessed ages range from 7 to 18 years) were contacted and invited to participate. We started with all participants whose parents had responded and correctly completed the CBCL ( $65 \%$ of all contacted births; $n=5468$ children). Participants were excluded if any of the 8 CBCL-OCS items were not completed. CBCL-OCS scores from each of these 5306 remaining participants were computed and the frequency tables were prepared. The mean age of boys was $9.0(\mathrm{SD}=1.3)$ for the $7-11$ age group and $15.1(\mathrm{SD}=1.9)$ for the $12-18$ age group. The mean age of girls was $8.9(\mathrm{SD}=1.2)$ for the $7-11$ age group and $15.0(\mathrm{SD}=1.8)$ for the $12-18$ age group.

Finally, in order to control for developmental differences in expression of CBCL-OCS symptoms in a general population twin sample, we analyzed data on 7-year-old and 10-year-old twins from the Netherlands Twin Registry. The characteristics of this sample are described elsewhere (Boomsma, 1998). The study is part of an ongoing twin-family study of health-related characteristics, personality, and behavior in the Netherlands, the Netherlands Twin Registry (NTR) (Boomsma, 1998). The NTR currently has data about more than 25,000 twin pairs from age 3 to 30. For this study, we assessed a sample of Dutch twin pairs whose parents (i.e., caregivers) reported on their behavior when the twins were 7 and 10 years old. Birth cohorts 1986 to 1992 participated in the 7-year assessment. The 10-year assessment included all birth cohorts from 1986 to 1994 . Thus, the sample of 10 -year-old twins presented here is the subset of the 7 -year-old sample who had reached age 10 by the time of the study. Parents were sent the CBCL and were asked to return it to the NTR by mail. Parents who did not return the forms within two months received a reminder, and persistent nonresponders were contacted by phone four months after the initial mailing. This procedure resulted in an average continued participation rate of $80 \%$. We started with a sample of 11,374 children aged 7 and 7124 children age 10. Participants were excluded if any of the 8 CBCL-OCS items were not completed. CBCL-OCS scores from each of the 10,194 remaining 7-year-old participants and the remaining 6448 10-year-old participants were computed and placed into frequency tables.

\section{Measures}

The CBCL is a standardized questionnaire used for parents to respond to 118 problem behaviors exhibited by their child over the previous 6 months. The parent responds along a 3-point scale with the code of 0 if the item is not true of the child, 1 for sometimes true, and 2 if the item is often true for the child. The characteristics and psychometric stability of the CBCL have been well established (Achenbach, 1991). The analyses performed here used the 1991 version of the CBCL, but the same items can be scored on the more recent 2001 version (Achenbach \& Rescorla, 2001).

The CBCL-OCS was developed using factor analysis on $11 \mathrm{CBCL}$ items which were thought to likely predict OCD (Nelson et al., 2001). Using a 1-factor model, 8 items were retained and were shown to have good internal consistency (Cronbach's alpha $=.84$ ). Those items retained are shown in Table 1, along with their CBCL item number. A numerical value for the CBCL-OCS scale is created by adding the scores on these 8 items, thus limiting the scale to a range of 0 to 16 .

\section{Analysis}

Because there was a relatively small range of values on the CBCL-OCS, we determined sensitivity and specificity at all levels of responding. Additionally, we determined the best cutpoint on the basis of maxim-

Table 1 Obsessive-Compulsive Scale (CBCL-OCS) items

\begin{tabular}{|c|c|c|}
\hline $\begin{array}{l}\text { CBCL item } \\
\text { number }\end{array}$ & CBCL item & $\begin{array}{l}\text { CBCL syndrome } \\
\text { on which } \\
\text { item is scored }\end{array}$ \\
\hline 9 & $\begin{array}{l}\text { Can't get his/her mind off } \\
\text { certain thoughts; obsessions }\end{array}$ & $\mathrm{TP}$ \\
\hline 31 & $\begin{array}{l}\text { Feels he/she might think } \\
\text { or do something bad }\end{array}$ & A/D \\
\hline 32 & Feels he/she has to be perfect & $\mathrm{A} / \mathrm{D}$ \\
\hline 52 & Feels too guilty & $\mathrm{A} / \mathrm{D}$ \\
\hline 66 & $\begin{array}{l}\text { Repeats certain acts } \\
\text { over and over; compulsions }\end{array}$ & TP \\
\hline 84 & Strange behavior & TP \\
\hline 85 & Strange ideas & $\mathrm{TP}$ \\
\hline 112 & Worries & A/D \\
\hline
\end{tabular}

Note: $\mathrm{A} / \mathrm{D}=$ Anxious $/$ Depressed, $\mathrm{TP}=$ Thought Problems. 
izing both sensitivity and specificity. We then applied this cutpoint to the distribution of scores in the 1991 CBCL sample and the 3 twin samples to determine prevalence.

\section{Results}

\section{Determining the cutoff points for the CBCL-OCS}

The ROC curve is shown in Figure 1 for OCD positive participants compared to clinical controls and general population controls. The AUC for the CBCL-OCS when compared to clinical controls was .88 $(95 \%$ $\mathrm{CI}=.81-.94)$. When compared to general population controls the AUC was $.96(95 \% \mathrm{CI}=.94-.99)$. The full range of sensitivities and specificities at various cutpoints are provided in Table 2 . At the best cutpoint of 5 , the sensitivity was $91.8 \%$ and the specificity was $67.2 \%$ when compared to clinical controls. One possible explanation for the false positives is that the clinical control participants had other anxiety disorders that were being captured by the OCS. To test this, we looked at the diagnostic profiles of the false positives compared to the true negatives to see if there was a difference in the distribution of diagnoses between these two groups. Of the 21 clinical controls with false positives at this cutpoint on the CBCL-OCS, 10 had ADHD, 9 had Any Mood Disorder, 7 had ODD or CD, 3 had Substance Abuse, 1 had Any Anxiety Disorder, 1 had PDD-NOS, 1 had enuresis, and 11 had 2 or more diagnoses. Testing for a difference in the diagnoses between those clinical control participants who were false positive and true negative was not significant by Fisher's Exact Test (FET).

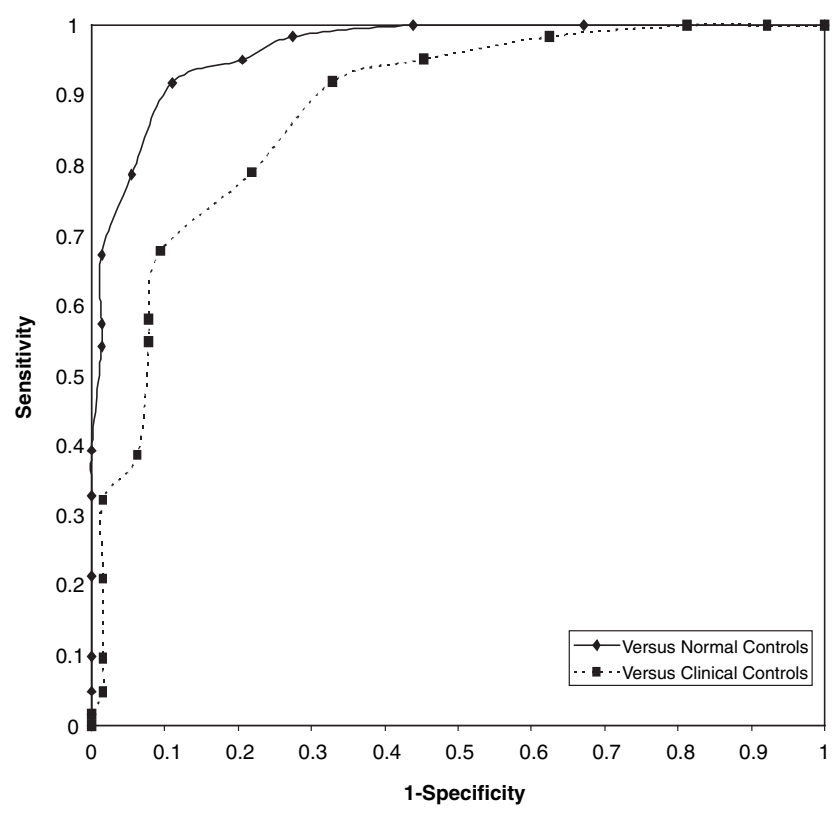

Figure 1 Receiver operating characteristic curve of the CBCL-OCS
Table 2 Sensitivity and specificity at all levels of the CBCLOCS. The best cutpoint to maximize sensitivity and specificity is indicated in bold

\begin{tabular}{rccc}
\hline & & \multicolumn{2}{c}{ Specificity } \\
\cline { 3 - 4 } Score & Sensitivity & $\begin{array}{c}\text { vs. general } \\
\text { population controls }\end{array}$ & $\begin{array}{c}\text { vs. clinical } \\
\text { controls }\end{array}$ \\
\hline 0 & 1.000 & .000 & .000 \\
1 & 1.000 & .329 & .078 \\
2 & 1.000 & .562 & .188 \\
3 & .984 & .726 & .375 \\
4 & .951 & .795 & .547 \\
$\mathbf{5}$ & .918 & .890 & .672 \\
6 & .787 & .945 & .781 \\
7 & .672 & .986 & .906 \\
8 & .574 & .986 & .922 \\
9 & .541 & .986 & .922 \\
10 & .393 & 1.000 & .938 \\
11 & .328 & 1.000 & .984 \\
12 & .328 & 1.000 & .984 \\
13 & .213 & 1.000 & .984 \\
14 & .098 & 1.000 & .984 \\
15 & .049 & 1.000 & .984 \\
16 & .016 & 1.000 & 1.000 \\
\hline
\end{tabular}

\section{Determining the percentages of CBCL-OCS positive patients in non-clinical samples}

After determining that the test had adequate sensitivity and specificity to be used as a tool to screen for OCD in both clinical and general population samples, the cutoffs were applied to the CBCL nonclinical normative sample and the 2 general population twin samples. The results are provided in Table 3. Looking first at the CBCL normative sample, using the best cutoff (CBCL-OCS score $\geq 5$ ) captured the top $6.0 \%$ of boys aged $4-11$ and $5.8 \%$ of boys aged $12-18$. This cutoff captured the top $5.9 \%$ of girls aged $4-11$ and $8.7 \%$ of girls aged $12-18$, a difference that neared significance (FET, $p=.072)$. There were no significant differences across age or sex in this group. The data from the Missouri twin sample show that in this population of children, a CBCL-OCS score of greater than or equal to 5 was observed in

Table 3 Percentage of children who are CBCL-OCS positive

\begin{tabular}{llc}
\hline & \multicolumn{2}{c}{$\begin{array}{c}\text { Percentage of sample } \\
\text { with CBCL-OCS } \geq 5\end{array}$} \\
\cline { 2 - 3 } Sample & Boys & Girls \\
\hline CBCL '91 normative sample & & \\
$\quad$ age 4-11 $(n=1196)$ & 6.0 & 5.9 \\
$\quad$ age 12-18 $(n=905)$ & 5.8 & 8.7 \\
Missouri twins & & \\
$\quad$ age 7-11 $(n=2715)$ & 7.1 & $4.9^{\mathrm{a}}$ \\
$\quad$ age 12-18 $(n=2591)$ & $4.3^{\mathrm{b}}$ & 4.0 \\
Dutch twins & & 2.3 \\
$\quad$ age 7 $(n=10194)$ & 2.4 & $3.8^{\mathrm{b}}$ \\
$\quad$ age 10 $(n=6448)$ & $3.3^{\mathrm{b}}$ & \\
\hline
\end{tabular}

Notes: a Sex difference significant at $p<0.05$ by Fisher's Exact Test. ${ }^{\mathrm{b}}$ Age difference significant at $p<0.05$ by Fisher's Exact Test. 
$7.1 \%$ of boys aged $7-11,4.3 \%$ of boys aged $12-18$, $4.9 \%$ of girls aged $7-11$ and $4.0 \%$ of girls aged $12-18$, slightly lower than the numbers seen in the CBCL sample. There was a difference across sex in only the younger sample (FET, $p=.019)$. The lower percentages seen in the older population of boys was significant (FET, $p=.001$ ). For the Dutch twin samples, the percentages are lower. In this population, a CBCL-OCS score of greater than or equal to 5 was observed in $2.4 \%$ of boys aged $7,3.3 \%$ of boys aged $10,2.3 \%$ of girls aged 7 and $3.8 \%$ of girls aged 10 . The higher percentage in the older population was significant for both age groups (FET, $p=.014$, and .000 for the younger and older groups, respectively). There was no significant difference across sex.

\section{Discussion}

\section{Clinical implications}

The results demonstrated that items contained in the CBCL can be used to screen patients for OCD. Using a cutoff score of 5 on the CBCL-OCS, we were able to identify over $91 \%$ of all DSM-determined cases of OCD in our clinical sample with reasonable specificity $(67.2 \%)$.

Identification of $2.4-7.1 \%$ of boys and $2.3-8.7 \%$ of girls in the general population with significant OCD symptoms in both general population and twin samples has implications for use of the CBCL-OCS as a screening tool in general pediatric populations as well as its usefulness for research into OCD.

In order for a screening tool to be useful, it must be accurate and should be used only if there is effectiveness of early detection (US Preventive Services Task Force, 1996). In addition, the cost of identifying an individual falsely (either positively or negatively) should not be so great as to outweigh the benefits of early detection of a person with the disease. In the case of the CBCL-OCS, we have shown that the test is accurate. What would be the cost to those individuals falsely identified? The positive score on the CBCL-OCS would lead to further questions on the part of the clinician in order to diagnose OCD (leading to treatment or further clinical evaluation) or it would lead the clinician into questions regarding other related disorders. Both of these two outcomes may be of considerable benefit. Finally, it has been shown that treatment of childhood OCD can improve clinical outcome (March \& Mulle, 1998), thus meeting the second criterion for the use of the CBCL-OCS as a screening tool.

Previous work on OCD in children has shown that prevalence increases markedly after the age of 13 (Heyman et al., 2001) with a shift in prevalence from being greater in males at a young age to affecting more females in adulthood, likely occurring after the age of 18 (Fireman, Koran, Leventhal, \& Jacobson, 2001). In the work reported here, we observed very little difference in the prevalence of CBCL-OCS determined symptoms between boys and girls, regardless of age or country of origin. One possibility is that the previous prevalence estimates used clinical samples where referral bias may lead to more boys than girls being identified in the clinic due to the over-representation of attentional and tic disorders in the clinic. Additionally, we have shown that there are little to no differences in the heritability of the CBCL-OCS between males and females (Hudziak et al., 2004). It is possible that the symptoms associated with OCD in young girls are considered normative or at least is not offensive enough to parents or teachers to lead to early identification. An analogous situation is inattentive problems as the primary manifestation of attention deficit hyperactivity disorder (ADHD) in females. We have previously argued that ADHD in females may be underdiagnosed as girls more often manifest the understudied severe attention problems subtype (Hudziak, 2001; Hudziak et al., 1998). However, gender effects may increase as the samples move into late adolescence. Future research will be directed at this important age range.

But what are the advantages to using this approach rather than a diagnostic interview or the DSM-IV criteria? The CBCL is easy for parents to complete, has normalized data stratified by age and gender, and has been cross-correlated with other instruments such as the Teacher Report Form (TRF) and the Youth Self Report (YSR) (Achenbach \& Rescorla, 2001). Currently, the CBCL is widely used and easy to incorporate into school assessments, well-child visits and academic evaluations. It has well-established relationships with DSM-IV childhood onset diagnoses, known reliability and stability of scores, is available in 69 languages with norms, has Teacher Report versions, and, as evidenced here, has large existing databases to determine cutoff scores for new scales in general pediatric populations.

For research purposes, the usefulness of the CBCL-OCS is considerable. Using the CBCL-OCS as a proxy for OCD may allow the researcher to screen in children who may have OCD or to screen them out of studies where comorbid OCD may be a problematic confound when looking at treatment response or epidemiological measures. Additionally, as we have shown here, there exist significant general population, twin, and family samples that have CBCL data available. Using the CBCL obtained in large twin populations it is possible to model obsessive-compulsive symtomatology along a continuum within the population (Hudziak, 2001). We have used this approach with populations of US twins of mixed age and ethnicity and Dutch twins, with combined samples that exceed 8000 twin pairs. We have shown that there are strong genetic and unique environmental factors accounting for clinical deviance on the CBCL-OCS in large twin populations (Hudziak et al., 2004). These data indicate signific- 
ant genetic contributions to, and no sex differences in, the OC syndrome.

\section{Limitations}

The sensitivity and specificity of the CBCL-OCS as a screening test were determined primarily by application of the tool in a post-hoc fashion to previously determined OCD cases. Ideally, one would like to perform a prospective trial using the CBCL-OCS in a general pediatrics population to select high and low risk groups to receive DSM interviews and to specifically control for comorbidity by using clinical controls matched for comorbid conditions - such as non-OCD anxiety disorders, which were over-represented in the OCD group presented here compared to the clinical controls. Additionally, one would like to know if identification of CBCL-OCS scores greater than or equal to 5 and subsequent further screening for OCD leads to improved outcome in children and, hopefully, a reduction of morbidity. Moreover, use of a case-control design as performed here has the limitation of possibly leading to 'spectrum bias.' Because the cases who were used in the experiment come primarily from tertiary referral sources, they may over-represent the stability and severity of the disorder and may make the measure less applicable to general population screening (Mower, 1999). Finally, the diagnosis of OCD was made on the basis of single skilled clinician assessment only. Using structured interviews with reliability estimates would have been preferable.

It is intriguing that the Dutch sample showed lower prevalence rates than the American samples. This could be for a number of reasons. 1) The American samples included older children than the Dutch samples. The peak age of onset of OCD for children has been thought to be in the teen years. Consequently, it is possible that the Dutch children simply have not yet come to an age where they would be showing signs and symptoms of OCD. 2) The CBCL-OCS cutoff determined from American samples is not as accurate for Dutch samples and we would need to re-norm the CBCL-OCS using a sample of children with OCD from the Netherlands (for example, if we used a cutpoint of greater than or equal to 4 in the Dutch twins we would capture 4.6$6.9 \%$ of children, similar to the American samples). 3) There is something inherently different about the American and Dutch OCD prevalence rates. This generally raises the question of the changes in OCD prevalence as measured by the CBCL-OCS as children age. Here, the stability of the findings in the Dutch twin samples at ages 7 and 10 are not completely surprising, because the 10 -year-old sample is a subset of the 7-year-old sample. However, there appears to be a trend in those samples of an increasing prevalence as the children age. A longitudinal examination of CBCL-OCS scores in Dutch twins from the NTR at ages 3, 7, 10, and 12 is under way and will provide more information about the development of OCD as measured by the CBCL-OCS.

\section{Conclusion}

Despite these limitations, it is still reasonable to use the CBCL-OCS as a tool for identifying OCD in the general clinical population. Given the lack of current clinical tools for cost-effective diagnosis, the high morbidity of the disease, the responsiveness of the disease to treatment, and the ease of parent-based screening with the CBCL, there are good reasons for using the CBCL-OCS in general pediatric and child and adolescent psychiatric practice.

\section{Correspondence to}

James J. Hudziak, University of Vermont, Department of Psychiatry, Given B229, Burlington, VT 05405, USA; Email: james/hudziak@uvm.edu

\section{References}

Achenbach, T.M. (1991). Manual for the Child Behavior Checklist/4-18 and 1991 Profile. Burlington, VT: University of Vermont Department of Psychiatry.

Achenbach, T.M., \& Rescorla, L.A. (2001). Manual for the ASEBA School-Age Forms \& Profiles. Burlington, VT: University of Vermont Research Center for Children, Youth, \& Families.

Berg, C.J., Rapoport, J.L., \& Flament, M. (1985). The Leyton Obsessional Inventory-Child Version. Psychopharmacology Bulletin, 21, 1057-1059.

Boomsma, D.I. (1998). Twin registers in Europe: An overview. Twin Research, 1, 34-51.

Calvocoressi, L., Lewis, B., Harris, M., Trufan, S.J., Goodman, W.K., McDougle, C.J., \& Price, L.H. (1995). Family accommodation in obsessive-compulsive disorder. American Journal of Psychiatry, 152, 441-443.

Costello, E.J., Angold, A., Burns, B.J., Stangl, D.K., Tweed, D.L., Erkanli, A., \& Worthman, C.M. (1996). The Great Smoky Mountains Study of Youth. Goals, design, methods, and the prevalence of DSM-III-R disorders. Archives of General Psychiatry, 53, 11291136.

Fireman, B., Koran, L.M., Leventhal, J.L., \& Jacobson, A. (2001). The prevalence of clinically recognized obsessive-compulsive disorder in a large health maintenance organization. American Journal of Psychiatry, 158, 1904-1910.

Flament, M.F., Whitaker, A., Rapoport, J.L., Davies, M., Berg, C.Z., Kalikow, K., Sceery, W., \& Shaffer, D. (1988). Obsessive compulsive disorder in adolescence: An epidemiological study. Journal of the American Academy of Child and Adolescent Psychiatry, 27, 764-71.

Geller, D.A., Coffey, B., Doyle, R., Shaw, B.A., \& Biederman, J. (2003). Validation of the ObsessiveCompulsive Scale of the CBCL. Proceedings of the 50th Anniversary Meeting of the American Academy of Child and Adolescent Psychiatry. 
Goodman, W.K., Price, L.H., Rasmussen, S.A., Mazure, C., Fleischmann, R.L., Hill, C.L., Heninger, G.R., \& Charney, D.S. (1989). The Yale-Brown Obsessive Compulsive Scale. I. Development, use, and reliability. Archives of General Psychiatry, 46, 1006-1011.

Grados, M., Scahill, L., \& Riddle, M.A. (1999). Pharmacotherapy in children and adolescents with obsessive-compulsive disorder. Child and Adolescent Psychiatric Clinics of North America, 8, 617-634, x.

Heyman, I., Fombonne, E., Simmons, H., Ford, T., Meltzer, H., \& Goodman, R. (2001). Prevalence of obsessive-compulsive disorder in the British nationwide survey of child mental health. British Journal of Psychiatry, 179, 324-329.

Hudziak, J.J. (2001). The role of phenotypes (diagnoses) in genetic studies of attention-deficit/hyperactivity disorder and related child psychopathology. Child and Adolescent Psychiatric Clinics of North America, 10, 279-297, viii.

Hudziak, J.J., Heath, A.C., Madden, P.F., Reich, W., Bucholz, K.K., Slutske, W., Bierut, L.J., Neuman, R.J., \& Todd, R.D. (1998). Latent class and factor analysis of DSM-IV ADHD: A twin study of female adolescents. Journal of the American Academy of Child and Adolescent Psychiatry, 37, 848-857.

Hudziak, J.J., van Beijsterveldt, C.E.M., Althoff, R.R., Stanger, C.S., Rettew, D., Nelson, E.C., Todd, R., \& Boomsma, D.I. (2004). Genetic and environmental contributions to childhood obsessive behavior: A cross-cultural twin study. Archives of General Psychiatry, 61, 608-616.

March, J., \& Mulle, K. (1998). OCD in children and adolescents: A cognitive behavioral treatment manual. New York: Guilford Press.

March, J.S., Franklin, M., Nelson, A., \& Foa, E. (2001). Cognitive-behavioral psychotherapy for pediatric obsessive-compulsive disorder. Journal of Clinical Child Psychology, 30, 8-18.

McConaughy, S.H., Stanger, C., \& Achenbach, T.M. (1992). Three-year course of behavioral/emotional problems in a national sample of 4- to 16-year-olds: I. Agreement among informants. Journal of the American Academy of Child and Adolescent Psychiatry, 31, 932-940.

Mower, W.R. (1999). Evaluating bias and variability in diagnostic test reports. Annals of Emergency Medicine, 33, 85-91.

Nelson, E.C., Hanna, G.L., Hudziak, J.J., Botteron, K.N., Heath, A.C., \& Todd, R.D. (2001). Obsessivecompulsive scale of the child behavior checklist: Specificity, sensitivity, and predictive power. Pediatrics, 108, E14.
Pauls, D.L., Alsobrook, J.P., 2nd, Goodman, W., Rasmussen, S., \& Leckman, J.F. (1995). A family study of obsessive-compulsive disorder. American Journal of Psychiatry, 152, 76-84.

Piacentini, J. (1999). Cognitive behavioral therapy of childhood OCD. Child and Adolescent Psychiatric Clinics of North America, 8, 599-616.

Rapoport, J.L., Inoff-Germain, G., Weissman, M.M., Greenwald, S., Narrow, W.E., Jensen, P.S., Lahey, B.B., $\&$ Canino, G. (2000). Childhood obsessive-compulsive disorder in the NIMH MECA study: Parent versus child identification of cases. Methods for the Epidemiology of Child and Adolescent Mental Disorders. Journal of Anxiety Disorders, 14, 535-548.

Rey, J.M., Morris-Yates, A., \& Stanislaw, H. (1992). Measuring the accuracy of diagnostic rests using receiver operating characteristics (ROC) analysis. International Journal of Methods in Psychiatric Research, 2, 39-50.

Rice, M.E., \& Harris, G.T. (1995). Violent recidivism: Assessing predictive validity. Journal of Consulting and Clinical Psychology, 63, 737-748.

Riddle, M. (1998). Obsessive-compulsive disorder in children and adolescents. British Journal of Psychiatry Supplement, 91-96.

Snider, L.A., \& Swedo, S.E. (2000). Pediatric obsessivecompulsive disorder. JAMA, 284, 3104-3106.

Steinhausen, H.C., Metzke, C.W., Meier, M., \& Kannenberg, R. (1998). Prevalence of child and adolescent psychiatric disorders: The Zurich Epidemiological Study. Acta Psychiatrica Scandinavica, 98, 262-271.

US Preventive Services Task Force (1996). Guide to clinical preventive services (2nd edn). Washington, DC: US Department of Health and Human Services.

Valleni-Basile, L.A., Garrison, C.Z., Jackson, K.L., Waller, J.L., McKeown, R.E., Addy, C.L., \& Cuffe, S.P. (1994). Frequency of obsessive-compulsive disorder in a community sample of young adolescents. Journal of the American Academy of Child and Adolescent Psychiatry, 33, 782-791.

Zohar, A.H. (1999). The epidemiology of obsessivecompulsive disorder in children and adolescents. Child and Adolescent Psychiatric Clinics of North America, 8, 445-460.

Zohar, A.H., Ratzoni, G., Pauls, D.L., Apter, A., Bleich, A., Kron, S., Rappaport, M., Weizman, A., \& Cohen, D.J. (1992). An epidemiological study of obsessive-compulsive disorder and related disorders in Israeli adolescents. Journal of the American Academy of Child and Adolescent Psychiatry, 31, 1057-1061.

Manuscript accepted 13 January 2005 